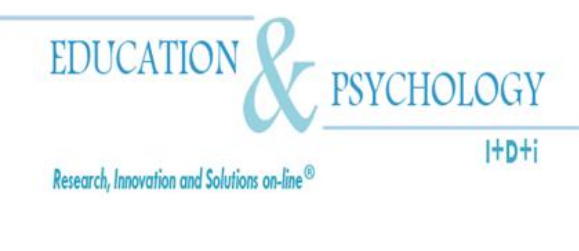

\title{
Motivar para Pensar Críticamente
}

\section{Sonia Olivares ${ }^{1}$, Carlos Saiz $^{1}$ y Silvia F. Rivas ${ }^{1}$}

${ }^{1}$ Departamento de Psicología Básica, Psicobiología y Metodología de las

Ciencias del Comportamiento,

Universidad de Salamanca, Salamanca.

\section{España}

Correspondencia: Sonia Olivares Moral. Facultad de Psicología, Avda. de la Merced 109-131, 37005. Salamanca, España. E-mail: soniaolivares@usal.es

(C) Education \& Psychology I+D+i and Editorial EOS (Spain) 


\section{Resumen}

Introducción. En este artículo presentamos los resultados ofrecidos a partir de una experiencia innovadora docente que fomenta el desarrollo de las habilidades de pensamiento crítico, a través de una intervención de naturaleza motivacional. Entendiendo el pensamiento crítico como una teoría de la acción, "pensamos para resolver problemas", y acompañando a esta concepción de un programa de enseñar/aprender a pensar y de metodologías activas como el aprendizaje basado en problemas (ABP), desarrollamos un programa paralelo que persigue fomentar la motivación para el desarrollo de dichas habilidades. Esta instrucción motivacional se sustenta en una opción teórica que entiende la motivación como el resultado de dos variables Expectativa/Valor. Estos dos conceptos posibilitan una intervención motivación en pensamiento crítico más eficaz.

Método. En este estudio participaron 89 universitarios españoles, con edades comprendidas entre los 20 y 30 años y de ambos sexos, quienes fueron evaluados mediante el PENCRISAL y el CMA tanto al inicio como al final de la intervención, junto con una evaluación continua. Durante dicha intervención, la totalidad de los alumnos recibieron una instrucción en pensamiento crítico. Sin embargo, sólo la mitad de la muestra participó además en un programa motivacional.

Resultados. Los resultados revelan que la instrucción en las habilidades de pensamiento crítico, a través de ARDESOS, ha sido exitosa. Además, los resultados obtenidos en motivación, han producido un mantenimiento de las puntuaciones en el grupo que disfrutó del programa motivacional.

Discusión. Los resultados muestran que la intervención motivacional ha funcionado en buena medida. Sin embargo, es necesario incrementar estas mejoras. De esta manera, se aporta así una herramienta para el estudio de los factores motivacionales del pensamiento crítico, el cual puede ser especialmente útil en la perspectiva de la instrucción y la intervención pedagógica.

Palabras Clave: pensamiento crítico; motivación; instrucción; evaluación. 


\title{
Encouragement for Thinking Critically
}

\begin{abstract}
Introduction. Here we report the results obtained in an innovative teaching experience that encourages the development of Critical Thinking skills through motivational intervention. Understanding Critical Thinking as a theory of action, "we think to solve problems", and accompanying this concept with a program aimed at teaching/learning Critical Thinking and with active methodologies such as Problem-Based Learning" (PBL), we developed a parallel program designed to foster motivation for the development of these skills. This motivational instruction is based on a theoretical option that understands motivation as the result of two variables: Expectancy/Value. These two concepts enable an intervention in motivation towards more efficient Critical Thinking.
\end{abstract}

Method. 89 Spanish university students of both sexes were enrolled in the study, with ages between 20 and 30 years, who were assessed by means of the PENCRISAL and the AGQ at both the start and the end of the intervention, together with continuous assessment. During the intervention, all the students received instruction in Critical Thinking. However, only half of the sample also participated in the motivation program.

Results. The results show that instruction in Critical Thinking skills through ARDESOS was successful. Moreover, the results obtained concerning motivation pointed to a maintenance of the scores in the group subjected to the motivation program.

Discussion. The results of the present study show that to a large extent intervention in motivation functions well. Nevertheless, it is necessary to increase such improvements. Thus, we describe a tool for studying the motivational factors of Critical Thinking, which could be especially useful from the perspective of instruction and pedagogical research.

Keywords: Critical thinking, motivation, instruction, assessment.

Received: 03/30/13 Initial acceptance: 04/18/13 Final acceptance: 07/19/13 


\section{Introducción}

Bien es sabido que para aprender es necesario ser capaz y estar dispuesto a ello. Ambos factores se precisan como necesarios. La teoría es atractiva, pero la realidad es bien distinta. También es conocido que, en las diferentes etapas educativas, un reto importante es conseguir que los estudiantes estén dispuestos a aprender, es decir, motivarles. Y este desafío no es fácil. En el caso que nos ocupa, el ámbito universitario, este hecho es aún más importante. Cabría preguntarnos qué acciones llevaríamos a cabo para lograr mejorar la motivación en nuestros universitarios a la hora de aprender, por qué se esfuerzan cuando estudian, qué buscan aprender o cómo estableceríamos las mejores condiciones para fomentar dicha motivación... Siendo así, lo ideal sería que el corazón del sistema educativo promocionara la exploración, el descubrimiento y el desafío cognitivo, que los contextos educativos brindaran novedad y complejidad, retos deseados, metas pretendidas y feedback personalizado, todo ello con el fin de alimentar la motivación intrínseca. Sin embargo, nos encontramos que este paradigma es una mera utopía en la mayoría de los casos.

En múltiples revisiones realizadas hasta la actualidad, han surgido muchos y muy variados programas que procuran nutrir la motivación de nuestros estudiantes en busca de una mejora en sus rendimientos académicos. Gran parte de dichas iniciativas son excesivamente generales y amplias, donde caben numerosas interpretaciones por parte de nuestros docentes de cómo llevar a cabo dichas intervenciones y trabajar este constructo con nuestro alumnado. Desde luego, pistas para promover la motivación tenemos, pero a la hora de la verdad, no resulta fácil aplicarlas. Además, se añade la circunstancia que, estos programas, se centran mayoritariamente en la educación obligatoria, dejando de lado a la población universitaria.

Teniendo en mente este contexto, esta grave problemática todavía se acentúa más dentro del campo de estudio en el que venimos trabajando desde hace ya unos años, las habilidades de pensamiento crítico. Para nosotros, el pensamiento crítico es una teoría de la acción, es razonar y decidir para resolver, obteniéndose así grandes beneficios como son un conocimiento deseable de la realidad, una mayor sabiduría. Por tanto, pensar críticamente no es sólo profundizar en el terreno del buen juicio y de la buena argumentación, sino que además, es necesario que nos ayude a resolver problemas o alcanzar nuestras metas, convirtiéndose así la argumentación en un medio, no como un fin (Saiz y Rivas, 2011). 
La mayoría de los teóricos en el campo (APA, 1990; Ennis, 1996; Facione, 2011; Halpern, 1998, 2003; Paul \& Elder, 2001) consideran que para poder llevar a cabo este tipo de pensamiento con eficiencia, además de poseer este conjunto de habilidades, se requiere de la intervención de otro tipo de componentes, como son el metaconocimiento y la motivación. Lo ilustramos en la siguiente figura.

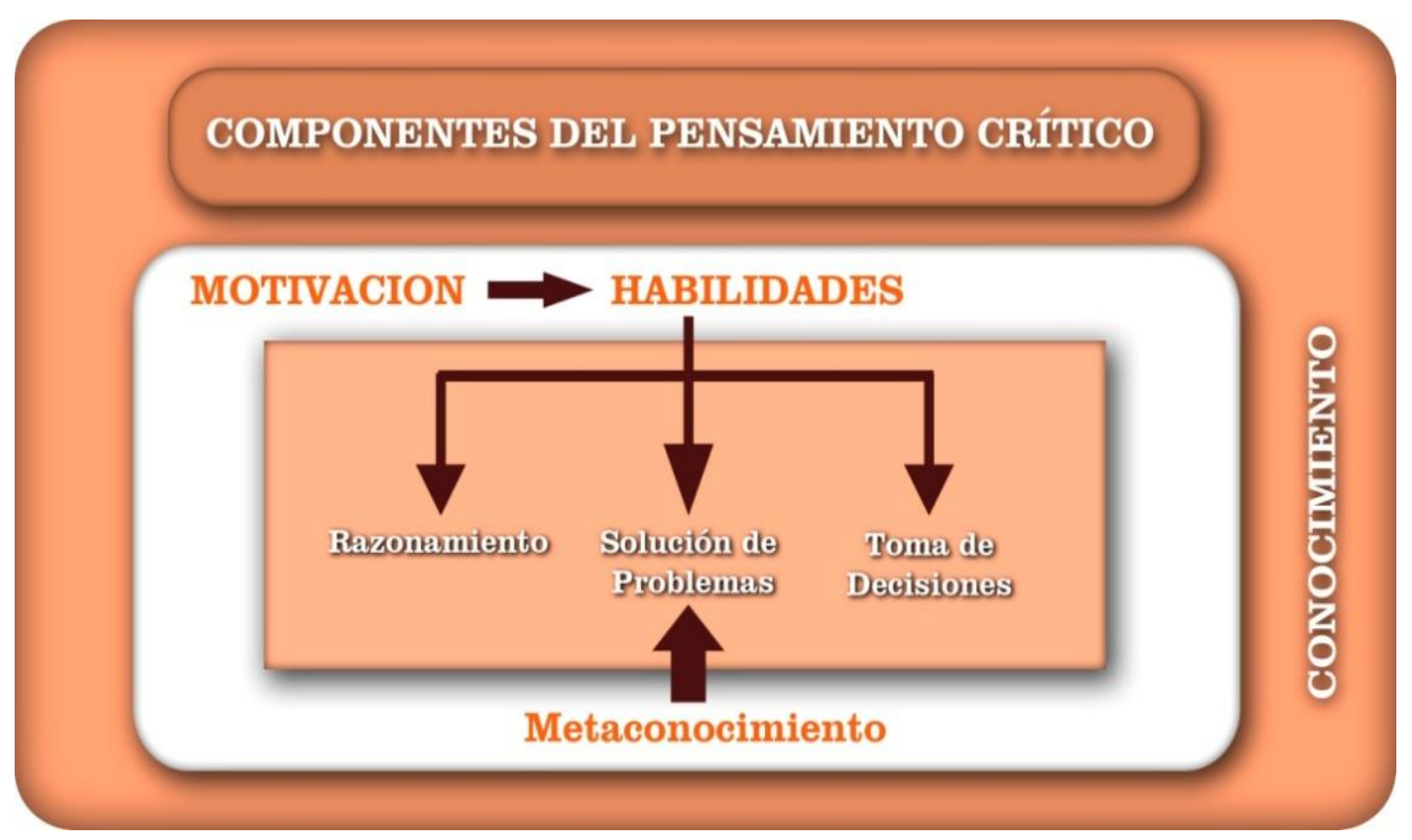

Figura 1. Componentes del Pensamiento Crítico (Saiz y Rivas, 2011).

Como podemos observar, el núcleo fundamental de los componentes del Pensamiento Crítico lo conforman las habilidades de razonamiento, solución de problemas y toma de decisiones. Sin embargo, la tenencia de estas habilidades no logra por sí sola una ejecución "eficaz", sino que se necesita que intervengan estos otros dos constructos. Para poder poner en marcha este tipo de pensamiento, necesitamos "querer" que suceda, necesitamos "desear" pensar críticamente. Aquí, es donde entra en juego la motivación. Ambos componentes, lo cognitivo (es decir, las habilidades) y lo motivacional o disposicional, son ineludibles, puesto que si una persona sabe qué habilidad debe de desplegar en una determinada situación, pero no está motivada para hacerlo o ejercitarlo, no será un buen pensador crítico. Igualmente, po- 
seer la disposición tampoco es suficiente, ya que si una persona está dispuesta y motivada a pensar críticamente, pero no sabe cómo hacerlo, tampoco lo logrará. Ambos componentes, habilidades y disposiciones, constituyen la esencia de este trabajo.

A su vez, necesitamos también del metaconocimiento para dirigir, organizar y planificar nuestras habilidades de una manera rentable, una vez que éstas han comenzado a funcionar. Como puede observarse, se trata de un factor importante a tener en cuenta. No obstante, en nuestra intervención lo mantenemos al margen, ya que está siendo tratado en otros trabajos realizados actualmente.

Con respecto a las habilidades del Pensamiento Crítico, es aceptado que representan el componente cognitivo, el saber qué y cómo hacer. Para la enseñanza y desarrollo de estas habilidades, se ha confeccionado un programa de instrucción llamado ARDESOS (ver Saiz y Rivas, 2011) el cual detallaremos brevemente más adelante. Por el contrario, en relación al componente disposicional, no existe tal claridad conceptual. En este contexto, nuestra opción es considerar la motivación desde la perspectiva del modelo Expectancy and Value propuesto por Eccles et al. (1983, 1987,1993; Eccles \& Wigfield, 2002; Wigfield, 1994; Wigfield \& Eccles, 1992, 2000), ya que nos ofrece grandes ventajas como una solidez teórica refrendada en múltiples investigaciones y la posiblidad de operacionalizar fácilmente sus variables de cara a la intervención. Estos modelos nacen de una perspectiva cognitiva general y reflejan la metáfora de que la persona es un tomador de decisiones activo y racional, lo cual encaja perfectamente dentro de nuestra tesis de Pensamiento Crítico. Es importante clarificar que estos autores concebían una motivación aplicada a la elección y el logro o rendimiento dentro de un dominio concreto (matemáticas). A pesar de ésto, y en los que a nosotros nos incumbe aquí, señalamos la Expectativa y el Valor como una forma específica de llevar a cabo una actividad. Asimismo, la motivación se focalizaría sobre el Pensamiento Crítico, definido en términos operativos para efectos de su medición, como el modo de pensar que se caracteriza por su rigor. 


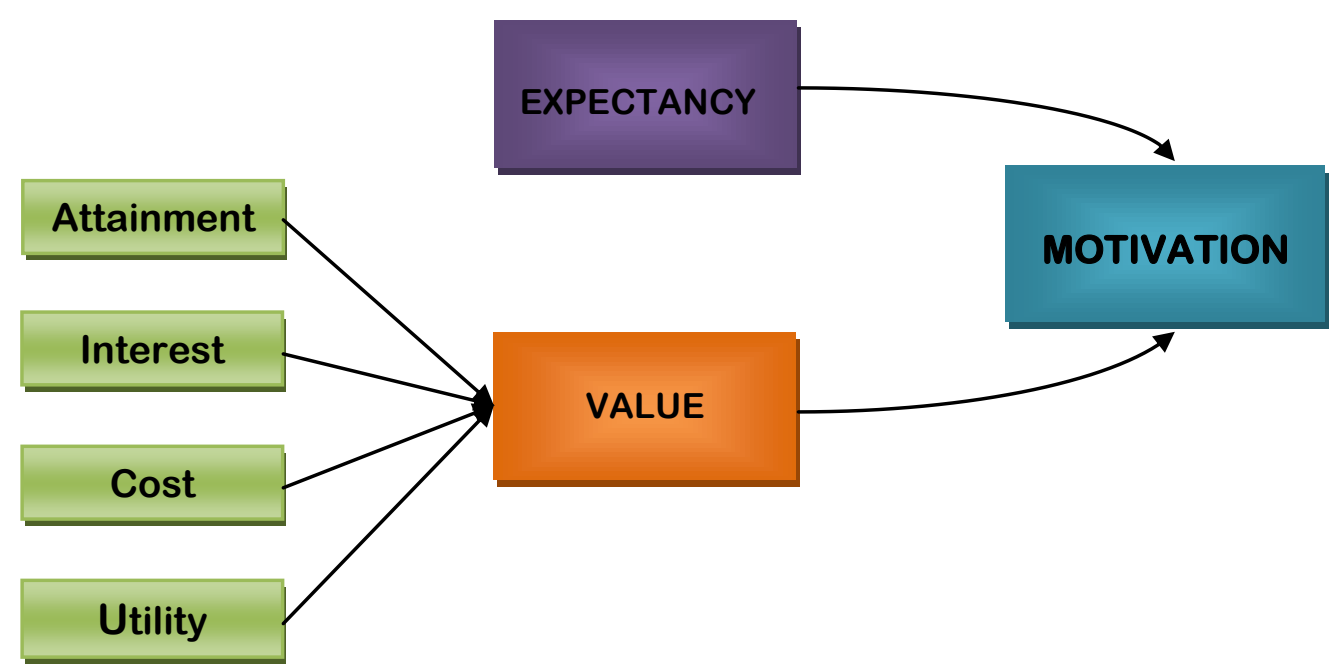

Figura 2. Modelo de motivación de Eccles y Wigfield (simplificado).

Como observamos en la figura, el componente Expectativa, hace referencia a las creencias de los estudiantes acerca de sus posibilidades de éxito en una tarea (en este caso, pensar de forma crítica en una situación). Incluye juicios de autoeficacia y control (Pintrich, 1989) y la confianza en las propias destrezas cognitivas para llevar a cabo una tarea académica en el futuro. El segundo componente del modelo es el Valor. Se refiere a la valía asignada a una tarea, aquí al valor que tiene para la persona el hecho de pensar críticamente. Este constructo está compuesto a su vez por cuatro factores: percepciones sobre cómo es de importante para la persona realizar bien una tarea (attainment); el interés intrínseco que tiene la tarea (interest); el coste que supone realizarla (cost); y la utilidad o instrumentalidad que ésta posee para metas futuras (utility) (Eccles et al., 1983; Wigfield \& Eccles, 1992).

En nuestro estudio, trabajamos estas variables de manera conjunta con el objetivo de mejorar las habilidades de Pensamiento Crítico, pero hacemos especial hincapié en el factor utilidad, debido a la transcendencia que éste tiene y a la carencia de datos en investigaciones sobre el mismo. Consideramos extraordinariamente necesario que los estudiantes sepan qué metas se pretenden conseguir en cada una de las actividades planteadas y para qué sirve aquello que van a aprender de tal manera que todo ésto facilite el poder mantener su entusiasmo e interés. Es crucial que conozcan cuál es el objetivo o propósito en la realización de cada una de las tareas académicas y sentir además, que ello cubre alguna necesidad personal (de informarse, de profundizar, de relacionar, de integrar, etc.). Lo que sucede es que si un alumno no conoce el propósito de una tarea y no puede relacionar dicho propósito con la comprensión de 
lo que la tarea implica y con sus propias necesidades, muy difícilmente va a poder llevar a cabo un estudio en profundidad y su posterior transferencia a la vida cotidiana. De lo contrario, al no poder relacionar la tarea con las finalidades de la misma, el alumno tenderá a adoptar un enfoque de tipo superficial para la consecución de la misma (Rinaudo, Barrera y Donolo, 2006). Estamos convencidos de que si desde el profesorado se proporciona a los estudiantes una clara indicación sobre los objetivos que se espera que alcancen, y se les hace ver los procesos que van utilizando o que pueden llegar a utilizar para la consecución de tales objetivos, ellos irán autorregulando sus recursos cognitivos. Para ello, incorporamos este factor en nuestra intervención, planteando la cuestión de si hay algo más importante que la reflexión crítica y mostrando sus bondades con resultados. Es decir, uno se interesa y se esfuerza por aprender aquellos contenidos que va a poner en práctica y le van a ser útiles para su vida. De esta manera, el aprendizaje que se lleva a cabo es mucho más profundo y significativo.

Según todo este planteamiento teórico aquí presentado, nuestra hipótesis de trabajo es que esperamos una mejora de las habilidades de Pensamiento Crítico después de la intervención, obteniéndose un mejor rendimiento en el grupo que reciba la instrucción en motivación. Igualmente, se pronostica un incremento en las puntuaciones de motivación, después de dicha intervención.

\section{Método}

\section{Participantes}

Debido a la complejidad de las habilidades de Pensamiento Crítico, el programa es dirigido a poblaciones que posean al menos un nivel intelectual medio. Por este motivo, se utilizó una muestra de 90 estudiantes de $4^{\circ}$ de Psicología de la Universidad de Salamanca (España), los cuales se encontraban cursando la asignatura de "Psicología del Pensamiento". Por diferentes razones (falta de información, test incompletos, etc.), nos encontramos con una mortalidad experimental de un sujeto. De esta manera, la muestra final utilizada se compuso de 89 casos, de los cuales un 91\% (81) fueron mujeres y un 9\% (8) varones. La media de edad de los participantes fue de 21,72 años con una desviación típica de 1,846 años. 


\section{Intrumentos}

PENCRISAL, test de Pensamiento Crítico. Con el fin de medir la magnitud del efecto y determinar así en cuál de los dos grupos se produce un mayor incremento en la mejora de las habilidades de Pensamiento Crítico se aplica el test PENCRISAL desarrollado por Saiz y Rivas (Rivas y Saiz, 2012; Saiz y Rivas, 2008). Se trata de una prueba que consta de 35 situaciones-problema de producción, con un formato de respuesta abierta y estructurado en torno a 5 factores: Razonamiento Práctico, Deducción, Inducción, Toma de Decisiones y Solución de Problemas $(\alpha$ de Cronbach $=.632$; test-retest: $r=.786$, Rivas y Saiz, 2010). Cada uno de estos factores albergan las estructuras más representativas consiguiendo de esta manera, recoger las habilidades fundamentales de pensamiento y las formas más relevantes de reflexión y resolución de nuestro funcionamiento cotidiano.

Para su aplicación, se optó por una versión informatizada y de manera colectiva, principalmente debido a las considerables ventajas que así se obtienen tanto para la corrección como para la propia ejecución. Para dicha corrección, están establecidos unos criterios de corrección estandarizados que asignan valores entre 0-2 puntos, según la calidad de la respuesta ofrecida por los sujetos. Y respecto a la ejecución, al tratarse de un test psicométrico de potencia, se brinda la posibilidad de realizarlo en diferentes sesiones para reducir posibles efectos del cansancio, por lo que no existe una limitación temporal. No obstante, la duración media estimada para completar la prueba es de 60-90 minutos. Para una información más detallada de la prueba puede consultarse Saiz y Rivas (2008b) y Rivas y Saiz (2012).

CMA, Cuestionario de Metas Académicas. Con el objetivo de determinar en cuál de los dos grupos se produce un incremento mayor en la mejora de la motivación académica, hemos aplicado el CMA (Hayamizu \& Weiner, 1991). Una descripción más detallada respecto a la traducción puede encontrarse en Núñez y González-Pienda (1994).

Este cuestionario toma medidas sobre qué tipo de tendencias motivacionales o factores tienen los estudiantes a la hora de dirigir sus esfuerzos hacia los logros académicos. La prueba está compuesta por 20 ítems con un formato de respuesta tipo Likert de 5 valores, donde la tarea simplemente consiste en señalar en qué grado cada una de las razones o motivos proporcionados en la escala, influye en la disposición del sujeto a la hora de estudiar. 
Estos 20 ítems se distribuyen en torno a tres factores motivacionales: Metas de Aprendizaje (factor I), Metas de Logro (factor II) y Metas de Refuerzo Social (factor III) ( $\alpha$ de Cronbach=.815). Es importante tener en cuenta que estos tres tipos de metas académicas no son mutuamente excluyentes y sólo expresan disposiciones, por lo cual un estudiante puede perseguir más de una de ellas. Nuevamente, para su administración elegimos la opción informatizada junto con la aplicación colectiva en diferentes aulas de la facultad de Psicología, por las ventajas que previamente hemos citado.

\section{Diseño}

Con el fin de analizar la eficacia de la intervención, se realizó un diseño cuasiexperimental, con medidas pre-post tratamiento, grupo de intervención y grupo de cuasicontrol equivalente. Efectuamos un muestreo intencional o de conveniencia, donde la mitad de la muestra fue seleccionada a efectos del grupo experimental y la otra mitad a efectos del grupo cuasi-control. De esta manera, ambos grupos recibieron por igual la instrucción en las habilidades de Pensamiento Crítico mediante el programa ARDESOS. Sin embargo, únicamente el grupo experimental obtuvo además la instrucción en motivación.

\section{Programa de intervención}

El método de trabajo que utilizamos para la intervención y que a continuación explicamos, recoge los dos aspectos imprescindibles para la mejora del Pensamiento Crítico comentados anteriormente: los componentes cognitivos (habilidades) y los componentes motivacionales (disposiciones). Como lo uno no es sin lo otro, es decir, ni las habilidades cognitivas ni las disposiciones por sí solas son suficientes para capacitar a una persona a pensar críticamente, la intervención diseñada incorpora por un lado, una consolidada metodología activa de aprendizaje llamada ARDESOS para la adquisición de las habilidades de pensamiento crítico (Saiz y Rivas, 2011), y por otro, un programa de acciones dirigidas a la supervisión y control del curso de la motivación de los alumnos, éste último, objeto de esta investigación.

\section{Programa ARDESOS}

Con el fin de buscar esa mejora de las habilidades intelectuales que constituyen el Pensamiento Crítico, nos servimos del programa ARDESOS (para más detalles, ver Saiz y Rivas, 2011). Este programa está basado en la enseñanza directa de habilidades a través de un instructor experto, habilidades que se constituyen como conocimientos de procedimientos, donde la intervención se centra principalmente en la retroalimentación del proceso de apren- 
dizaje llevado a cabo, más que en el propio aprendizaje de puros contenidos. La estrategia de enseñanza-aprendizaje es el Aprendizaje Basado en Problemas (ABP), mediante problemas cotidianos. Esta metodología promueve los procesos metacognitivos y permite a los estudiantes la práctica con situaciones reales, donde deben buscar e investigar sus propias respuestas y soluciones antes de llevarse a cabo la instrucción en el aula, lo cual nos ofrece cruciales ventajas para el éxito del programa. En las sesiones se pretende que los estudiantes resuelvan las situaciones problemáticas activamente $\mathrm{y}$, posteriormente, se facilita un análisis detallado y se efectúa una evaluación de su trabajo, la cual se completa con la discusión en clase por el instructor de todas las dificultades y las dudas que hayan surgido y una clarificación de estos problemas. Por tanto, la evaluación es continua.

Para el trabajo de los cinco bloques de contenidos, a saber, razonamiento deductivo, razonamiento inductivo, razonamiento práctico, toma de decisiones y solución de problemas, se dispone de 20-30 horas presenciales, distribuidas en 15-20 sesiones semanales de noventa minutos y un tiempo máximo de 60 horas, incluyendo el propio trabajo de los estudiantes.

Respecto a las clases de grupo grande (50-60 alumnos), se trabajan todos los conceptos de la materia siempre partiendo de las actividades realizadas previamente por los alumnos y mediante los procedimientos de capacitación, reflexión y discusión por parte del instructor, junto con la estrategia ABP, para la adquisición de las competencias en Pensamiento Crítico. Las clases de grupos pequeños están organizadas en seminarios con un máximo de quince alumnos por grupo y en subgrupos de tres personas cada uno, de forma que se lleve a cabo un aprendizaje y trabajo cooperativo. En estas clases, es donde los alumnos exponen los trabajos que han ido realizando a lo largo de la semana, y donde se facilita otro espacio para la discusión y reflexión sobre todos los aspectos acontecidos, y siempre modelado por el instructor.

\section{Programa de Ayudas Motivacionales}

Para llevar a cabo esta intervención en motivación establecemos, por una parte, un programa de tutorías $y$, por otra, un seguimiento a lo largo de clases de grupo pequeño sobre las intervenciones y el grado de implicación de los diferentes estudiantes en las mismas.

Antes de proseguir con la explicación, queremos enfatizar que todo este programa se ofrece a los alumnos como un conjunto de ayudas en su desempeño en la materia. De tal manera que dichos apoyos, trabajen sobre la motivación de los estudiantes a través de acciones 
que faciliten la búsqueda de utilidad de los contenidos a aprender y, con ello, facilitar también la transferencia de los mismos a sus vidas diarias de una manera integrada. Por tanto, se trata de proveer un acompañamiento a los alumnos a lo largo de la instrucción, el cual proporciona un apoyo ante las dificultades que puedan surgir y una facilitación de los usos prácticos de las habilidades de pensamiento crítico de forma integrada.

Para una mejor lectura y seguimiento de este programa de tutorías, ofrecemos a continuación un esquema de las diferentes partes de las que consta dicha intervención:

- Tutorías grupales de inicio de bloque.

- Registro de conductas en las clases de grupo pequeño y cuadernillo de reflexiones sobre las prácticas.

- Tutorías de seguimiento o tutorías de grupo de trabajo.

- Tutorías grupales de cierre de bloque.

\section{Tutorías Grupales de Inicio de Bloque.}

Este tipo de tutorías se realiza siempre al comienzo de cada uno de los bloques de contenidos y se llevan a cabo en grupos de quince alumnos. Al haber tres grupos de quince alumnos cada uno y, teniendo en cuenta que trabajamos cinco bloques de contenidos, a lo largo de todo el estudio el instructor en motivación lleva a cabo quince tutorías grupales de inicio de bloque. El objetivo que pretenden estas tutorías es la realización de una introducción de los contenidos de manera aplicada a diferentes contextos, sin tocar ningún aspecto teórico de los contenidos y siguiendo un guión previamente establecido para procurar una intervención sistemática entre los diferentes grupos. Cada sesión se lleva a cabo durante 20-30 minutos y siempre antes de que el bloque correspondiente comience en las clases de grupo grande bajo una programación temporal establecida dentro del calendario de la asignatura.

El esquema general de actuación se detalla en lo siguiente:

1.- Toma de contacto con los alumnos al inicio de cada uno de los bloques de contenidos. Cuando se trata de la primera tutoría grupal de toda la intervención, se lleva a cabo una explicación de la dinámica a seguir con el programa de motivación para ofrecerles una orientación global del proceso a seguir. 
2.- Exploración de los conocimientos previos de los estudiantes según el bloque de contenidos que corresponda (¿qué haces cuando...?, ¿cómo te afrontas a la situación de...?, ¿cómo te hubiera gustado actuar en el momento de...?) y aportación del feedback conveniente.

3.- Síntesis de los comentarios y su re-enfoque hacia la utilidad a través de las metas y submetas de trabajo.

4.- Clarificaciones y recomendaciones hacia la importancia de la adaptabilidad y flexibilidad de estándares de actuación.

5.- Resumen clarificador y recordatorio de las tareas a desarrollar por parte de los alumnos en cada bloque. Cierre de la tutoría grupal.

Con esta serie de actuaciones, se pretende facilitar la iniciación al bloque de contenidos para las posteriores clases de grupo grande. De este modo, proporcionamos a los alumnos una primera toma de contacto con las habilidades esenciales del Pensamiento Crítico, buscando con ello promover un aprendizaje significativo de cara a la utilidad y una puesta en práctica de los contenidos que se desarrollarán en las clases posteriores.

Registro de Conductas en las clases de grupo pequeño y Cuadernillo de Reflexiones sobre las Prácticas.

Una vez que hemos llevado a cabo la tutoría grupal de inicio de bloque, el siguiente momento en la intervención se constituye en la realización de un registro de las conductas en las clases de grupo pequeño. Cada grupo de quince alumnos recibe una clase práctica a la semana, la cual tiene una duración de noventa minutos donde el objetivo que se persigue es el de exponer y debatir las tareas realizadas por el grupo de trabajo.

En cada una de estas clases, el instructor en motivación es un mero observador y simplemente realiza un registro de los comentarios efectuados por cada alumno del tipo: grado de intervención en la práctica, si realiza o no intervenciones espontáneas, número de estas intervenciones, tipo y número de preguntas que efectúa al profesor (preguntas conceptuales o de modelo de situación) y si realiza preguntas a sus compañeros y tipo. Toda esta información recabada nos sirve de retroalimentación para el ajuste de las intervenciones en las tutorías de seguimiento y nos permite un seguimiento de si se producen o no cambios en las intervenciones de los alumnos en las clases prácticas. 
Al finalizar cada una de las prácticas, se indica a los alumnos que, de forma individual, respondan a la pregunta de utilidad sobre las habilidades trabajadas durante la sesión (¿Qué utilidad tiene para ti, en tu vida diaria, todo lo que hemos visto hoy en la clase de grupo pequeño?) en sus cuadernillos de reflexiones sobre las prácticas, en su bitácora. Se trata de una escueta anotación donde reflejen el/los uso/s personales que le dan en sus vidas cotidianas a las habilidades de Pensamiento Crítico que están trabajando, para después debatirlo en las tutorías de seguimiento, tanto con el instructor en motivación como con el resto de compañeros del grupo y así intercambiar impresiones, aprender nuevas utilidades, autorregular conductas, etc. Igualmente, en su bitácora también se sugiere que vayan añadiendo otras anotaciones que consideren relevantes para ser desarrolladas en las tutorías de seguimiento, de forma que cada alumno confeccione una bitácora con el fin de que, a medida que se vayan cerrando los bloques, se procure un barrido de la trayectoria seguida por cada uno de ellos y tomar conciencia de los pasos seguidos y destacar los logros alcanzados (feedback).

\section{Tutorías de Seguimiento o Tutorías de Grupo de Trabajo.}

Este tipo de tutoría se realiza con cada subgrupo formado por tres alumnos que ejecutan las tareas semanalmente a lo largo de la asignatura. Se efectúan durante la semana que queda disponible entre la clase de grupo pequeño y la siguiente, por lo que a lo largo del cuatrimestre se realizan un total de doce tutorías de seguimiento para cada uno de los quince grupos de trabajo. La duración aproximada de cada una de ellas es de unos 10 a 15 minutos.

En estas sesiones minigrupales, pretendemos efectuar una monitorización y un seguimiento del rendimiento del grupo de estudiantes respecto a las aplicaciones que realizan de las habilidades de Pensamiento Crítico en sus vidas cotidianas, así como las dificultades que encuentran al poner en marcha dicho proceso. Todo ello siempre de cara a la promoción de sus motivaciones.

Estas tutorías se llevan a cabo según unos protocolos pre establecidos con vistas a facilitar una intervención sistemática en los diferentes grupos de alumnos.

Simultáneamente, todos los datos son recogidos en una plantilla. El esquema de trabajo sería el siguiente:

\section{Breve introducción a la sesión.}


2. Exploración esfuerzo vs. motivación: en cada tutoría se pretende indagar cómo ha transcurrido el proceso de elaboración y exposición del trabajo semanal, cómo los alumnos han superado las dificultades acontecidas y la valoración sobre la cantidad de esfuerzo que han depositado en todo ello, con el fin de monitorizar el rendimiento del grupo de trabajo y trabajar el proceso de la puesta en marcha de las habilidades. Además, contaremos con la información facilitada por el registro de conductas realizado en las clases de grupo pequeño que nos sirve, como apuntamos anteriormente, de reajuste y retroalimentación para la intervención durante la sesión. Paralelamente, trabajamos con los alumnos los aspectos motivacionales de forma individual y diferencial, buscando la promoción del uso de dichas habilidades. Aquí, resulta elemental considerar que cada individuo es único y particular y que todas las intervenciones motivacionales no funcionan de la misma forma con todas las personas. De esta manera, facilitamos el acompañamiento personal durante toda la intervención, procurando una regulación y control sobre las creencias de agencialidad personal de nuestros alumnos, de sus emociones y de sus metas hacia el dominio y utilidad de las habilidades de Pensamiento Crítico. En estas sesiones es imprescindible crear un entorno sensible a nuestro alumno y que proporcione confianza, desafío y apoyo. Para ello, utilizamos diferentes estrategias según las demandas y circunstancias en las que se encuentren nuestros alumnos en cada sesión. En general, las estrategias de mayor uso serían: aportación de un feedback reiterado; empatizar con las dificultades encontradas en la realización de tareas; explicitación del bagaje de conocimientos previos al inicio de la asignatura; explicitación de la disposición al aprendizaje de los contenidos dentro de la asignatura; promoción de la reflexión y participación contínua en el desarrollo de los bloques; promoción de la observación y análisis de los comportamientos respecto al uso de las habilidades de Pensamiento Crítico de uno mismo y de los demás; explicación de la metodología de trabajo siempre que el alumno señale incertidumbre y desorientación; apoyo emocional y promoción de la percepción de autoeficacia y autocontrol; exposición de casos prácticos y ejemplos cotidianos de la aplicación de las habilidades de pensamiento crítico y propuestas de resolución de situaciones; enfatización de la integración de habilidades para su puesta en marcha en contextos diarios.

3. Pregunta de utilidad: se demanda a los alumnos que comenten las anotaciones que han realizado en su cuaderno de reflexión sobre las prácticas a lo largo de la semana. A medida que cada alumno comenta las utilidades que le ha otorgado a los contenidos vistos, se incita al debate y reflexión de las mismas con los compañeros del grupo de trabajo. Todas las aportaciones se refuerzan positivamente $\mathrm{y}$, a su vez, vamos desplegando y analizando ejemplos cotidianos variados que estén en consonancia con lo previamente comentado por el 
estudiante. De esta manera, se pretende favorecer la transferencia de las habilidades aprendidas a los contextos más cercanos de nuestros alumnos.

4. Metas de utilidad: trabajamos las submetas de aproximación para la puesta en marcha y uso de las habilidades de Pensamiento Crítico en la vida diaria. La idea es que los alumnos realicen un proceso de concreción de metas acoplado a su idiosincrasia para alcanzar un buen dominio de las habilidades del Pensamiento Crítico. Para facilitarlo, se les encomienda una "tarea de la semana", tareas que dependerán según el ritmo de trabajo del grupo en concreto y cuyo objetivo es provocar esfuerzos metacognitivos respecto a cómo aprenden, cómo aplican y cómo pueden mejorar su competencia, además de continuar facilitando la transferencia al mundo cotidiano. El enfoque que damos a estas tareas para la consecución de las submetas hacia la utilidad es el siguiente:

1) Reconocimiento e identificación del uso y práctica-reflexión de las habilidades de Pensamiento Crítico en su contexto más cercano, tanto académico como fuera del mismo (medios de comunicación, textos académicos, noticias, debates, amigos, familiares, etc).

2) Reconocimiento-reflexión del uso y práctica de las habilidades de Pensamiento Crítico en uno mismo.

3) Identificación y evaluación de la práctica de las habilidades de Pensamiento Crítico en el contexto.

4) Identificación y evaluación de la práctica de las habilidades de Pensamiento Crítico en uno mismo.

5) Mejora de la puesta en marcha y rendimiento del uso de las habilidades de Pensamiento Crítico en los demás y en un mismo.

5. Cierre de la tutoría: se realiza una pequeña síntesis explicitando de manera concisa las utilidades de las habilidades que han comentado los alumnos y poniendo énfasis en la importancia de esforzarse por detectarlas y aplicar dichas habilidades en situaciones cotidianas. Como punto final, se propone la siguiente meta a alcanzar y se anima a la práctica de las habilidades del bloque en cuestión.

\section{Tutorías Grupales de Cierre de Bloque.}

Estas tutorías tienen lugar una vez que se ha finalizado el bloque de contenidos en cuestión. Igualmente se efectúan en grupos de 15 alumnos, por lo que se realizan cinco tutorías grupales de cierre de bloque para un mismo grupo y un total de 15 tutorías grupales de cierre de bloque a desarrollar por parte del instructor en motivación. Cada una de ellas tiene 
una duración de 20-30 minutos. El fin perseguido por estas tutorias es la realización de una síntesis de todos los contenidos "prácticos" ofrecidos por los diferentes grupos de alumnos y que han sido debatidos a lo largo de las sesiones. Cada clausura de bloque se efectúa siguiendo unos protocolos pre establecidos para facilitar primero, una intervención sistemática en los diferentes grupos de alumnos y, segundo, para realizar una recopilación de todo lo relevante debatido en las sesiones, de manera que todos los grupos al terminar el bloque correspondiente, tengan disponible la misma información procedente tanto de las tutorías grupales de inicio de bloque, como de las de tutorías de seguimiento.

Asimismo, el esquema general de actuaciones en las tutorías grupales de cierre de bloque es el que sigue:

1) Breve introducción donde se indaga sobre el desarrollo del bloque y las dificultades que se les han planteado.

2) Recapitulación respecto a las utilidades trabajadas de las habilidades de pensamiento crítico vistas en el bloque en cuestión. Reflexión y autoevaluación sobre el trayecto realizado y metas alcanzadas.

3) Sintetización de la información procurada y aportación del feedback correspondiente a las intervenciones de los alumnos.

4) Integración de las utilidades del bloque trabajado con respecto al resto de habilidades vistas en los bloques anteriores.

5) Promoción de la autoreflexión y transferencia de las habilidades a la vida cotidiana.

\section{Procedimiento}

La aplicación de esta iniciativa docente se realizó a lo largo de un semestre en las aulas de la facultad de Psicología de la Universidad de Salamanca. Una semana antes del comienzo de la instrucción aplicamos tanto la prueba PENCRISAL como el test CMA a todos los estudiantes. De igual manera se procedió una semana después de la finalización de dicha intervención, con el fin de recoger la segunda medida de las variables. El tiempo transcurrido entre la medida pre-tratamiento y la medida post-tratamiento fue de cuatro meses. La intervención fue llevada a cabo por dos instructores.

\section{Análisis Estadístico}

Para verificar el grado de ajuste al modelo normal de Gauss se realizó un estudio exploratorio y descriptivo de todas las variables de medida a través de herramientas habituales, diagramas de caja y pruebas de bondad de ajuste de Kolmogorov-Smirnov. Una vez compro- 
bado ésto, se llevó a cabo la prueba ANOVA para examinar el efecto de la iniciativa y comprobar así si existían diferencias significativas entre las situaciones pre y post intervención. El tratamiento de los datos se llevó a cabo mediante el paquete estadístico SPSS (versión 18.0).

\section{Resultados}

Primeramente, se procedió a un análisis descriptivo de las propiedades de la muestra según los momentos de medida pre-post, tanto a nivel global (muestra total), como por grupos de intervención. Posteriormente, se llevó a cabo la prueba t de Student y los análisis del ANOVA.

Respecto a la descriptiva de todas las variables en las diferentes condiciones del estudio, comprobamos que algunas de ellas no se distribuían según la curva normal debido a que presentaban unas distribuciones con índices de asimetría y curtosis marcadamente negativos y lepticúrticos respectivamente.

Tabla 1: Comparación de las medias pre en Pensamiento Crítico total y motivación y sus factores correspondientes en función de los grupos de intervención.

\begin{tabular}{|c|c|c|c|c|c|c|c|c|}
\hline \multirow[b]{2}{*}{ Variables } & & \multirow[b]{2}{*}{$\mathbf{n}$} & \multirow[b]{2}{*}{ Media } & \multirow{2}{*}{$\begin{array}{l}\text { Desviación } \\
\text { Típica }\end{array}$} & \multirow{2}{*}{$\begin{array}{c}\text { Diferencia } \\
\text { entre medias }\end{array}$} & \multicolumn{3}{|c|}{ Prueba T Student } \\
\hline & & & & & & Valor T & g.l. & $\begin{array}{c}\text { p-sig. } \\
\text { (bilateral) }\end{array}$ \\
\hline $\begin{array}{l}\text { Pensamiento } \\
\text { Crítico Total }\end{array}$ & $\begin{array}{l}\text { g.e. } \\
\text { g.c. }\end{array}$ & $\begin{array}{l}44 \\
45\end{array}$ & $\begin{array}{l}25.32 \\
24.22\end{array}$ & $\begin{array}{l}6.15 \\
7.15\end{array}$ & 1.10 & .774 & 87 & $.441^{\mathrm{NS}}$ \\
\hline $\begin{array}{l}\text { Razonamiento } \\
\text { Práctico }\end{array}$ & $\begin{array}{l}\text { g.e. } \\
\text { g.c. }\end{array}$ & $\begin{array}{l}44 \\
45\end{array}$ & $\begin{array}{l}5.43 \\
4.96\end{array}$ & $\begin{array}{l}2.13 \\
2.52\end{array}$ & .47 & .960 & 87 & $.340^{\mathrm{NS}}$ \\
\hline $\begin{array}{l}\text { Razonamiento } \\
\text { Deductivo }\end{array}$ & $\begin{array}{l}\text { g.e. } \\
\text { g.c. }\end{array}$ & $\begin{array}{l}44 \\
45\end{array}$ & $\begin{array}{l}3.57 \\
3.56\end{array}$ & $\begin{array}{l}2.23 \\
1.96\end{array}$ & .01 & .028 & 87 & $.977^{\mathrm{NS}}$ \\
\hline $\begin{array}{l}\text { Razonamiento } \\
\text { Inductivo }\end{array}$ & $\begin{array}{l}\text { g.e. } \\
\text { g.c. }\end{array}$ & $\begin{array}{l}44 \\
45\end{array}$ & $\begin{array}{l}4.16 \\
3.87\end{array}$ & $\begin{array}{l}1.75 \\
1.68\end{array}$ & .29 & .802 & 87 & $.425^{\mathrm{NS}}$ \\
\hline $\begin{array}{l}\text { Toma de } \\
\text { Decisiones }\end{array}$ & $\begin{array}{l}\text { g.e. } \\
\text { g.c. }\end{array}$ & $\begin{array}{l}44 \\
45\end{array}$ & $\begin{array}{l}5.18 \\
5.33\end{array}$ & $\begin{array}{l}1.96 \\
1.97\end{array}$ & -.15 & -.362 & 87 & $.718^{\mathrm{NS}}$ \\
\hline $\begin{array}{l}\text { Solución de } \\
\text { Problemas }\end{array}$ & $\begin{array}{l}\text { g.e. } \\
\text { g.c. }\end{array}$ & $\begin{array}{l}44 \\
45\end{array}$ & $\begin{array}{l}7.07 \\
6.67\end{array}$ & $\begin{array}{l}2.89 \\
2.45\end{array}$ & .40 & .706 & 87 & $.482^{\mathrm{NS}}$ \\
\hline $\begin{array}{l}\text { Motivación } \\
\text { Total }\end{array}$ & $\begin{array}{l}\text { g.e. } \\
\text { g.c. }\end{array}$ & $\begin{array}{l}44 \\
45\end{array}$ & $\begin{array}{l}65.66 \\
66.67\end{array}$ & $\begin{array}{l}6.00 \\
5.45\end{array}$ & -1.01 & -.829 & 87 & $.409^{\mathrm{NS}}$ \\
\hline $\begin{array}{l}\text { Metas de } \\
\text { Aprendizaje }\end{array}$ & $\begin{array}{l}\text { g.e. } \\
\text { g.c. }\end{array}$ & $\begin{array}{l}44 \\
45\end{array}$ & $\begin{array}{l}29.93 \\
31.38\end{array}$ & $\begin{array}{l}4.08 \\
3.32\end{array}$ & -1.45 & -1.832 & 87 & $.070^{\mathrm{NS}}$ \\
\hline $\begin{array}{l}\text { Metas de } \\
\text { Logro }\end{array}$ & $\begin{array}{l}\text { g.e. } \\
\text { g.c. }\end{array}$ & $\begin{array}{l}44 \\
45\end{array}$ & $\begin{array}{l}26.00 \\
25.62\end{array}$ & $\begin{array}{l}3.58 \\
3.29\end{array}$ & .38 & .518 & 87 & $.606^{\mathrm{NS}}$ \\
\hline $\begin{array}{c}\text { Metas de } \\
\text { Refuerzo Social }\end{array}$ & $\begin{array}{l}\text { g.e. } \\
\text { g.c. }\end{array}$ & $\begin{array}{l}44 \\
45\end{array}$ & $\begin{array}{l}9.73 \\
9.67\end{array}$ & $\begin{array}{l}3.22 \\
3.00\end{array}$ & .21 & .092 & 87 & $.927^{\mathrm{NS}}$ \\
\hline
\end{tabular}

$\mathrm{NS}=$ correlacción no significativa $(\mathrm{p}>.50) \quad *$ Significativo al .05 $* *$ Altamente significativo .01 
Seguidamente, se llevó a cabo la prueba ANOVA donde comparamos las medidas prepost y los grupos entre sí, en todas las medidas analizadas. Asimismo, se calculó el efecto del cambio en cada caso. Primeramente, se efectuó un análisis de los supuestos de normalidad y homogeneidad de varianzas de la muestra. El Test de Kolmogorov-Smirnov arrojó que la muestra en la medida pre mantenía una distribución normal con $p<.05$ en todas las variables del estudio exceptuando en los factores de razonamiento inductivo $(p<.00)$ y metas de refuerzo social $(p<.01)$, donde mostró un ligero desvío de la curva normal pero no lo advertimos como importante, ya que la $p$ aún siendo $<.05$, es todavía $\geq .01$. Sin embargo, en la condición post, encontramos que, además de razonamiento inductivo y metas de refuerzo social, los factores de metas de aprendizaje y metas de logro tampoco cumplieron el principio de normalidad, obteniendo $p<.05\left(p_{\text {RIpost }}=.04 ; \mathrm{p}_{\text {MApost }}=.00 ; p_{\text {MLpost }}=.00 ; p_{\text {MRSpost }}=.00\right)$. El resto de variables, sí lo cumplieron. Respecto al principio de homogeneidad, el contraste de Levene reveló que se cumplía dicha condición en todas y cada una de las variables del estudio.

Los resultados del ANOVA nos muestran que, en relación a la variable Pensamiento Crítico total, en cuanto al momento de medida pre-post, sí se dan diferencias significativas con $p<.05\left(F_{P C}(1,82)=78.27, p=.00\right)$, donde la condición post obtiene un rendimiento mayor $\left(M_{\text {pre }}=24,77 ; M_{\text {post }}=33,72\right)$. Al igual que también ocurre a nivel intergrupal $p<.05\left(F_{P C}\right.$ $(1,82)=4,25, p=.04)$, el grupo experimental posee una media significativamente mayor $\left(M_{g e}=30.38\right)$ que el grupo control $\left(M_{g c}=28.11\right)$. Sin embargo, con relación a la intersección entre el grupo y el momento de la aplicación, no existen diferencias significativas $p>.05$ $\left(F_{P C}(1,82)=1.35, p=.24\right)$ (Véase tabla 2$)$.

Tabla 2: Resumen de la significación de los efectos de ambos factores sobre la variable Pensamiento Crítico total.

\begin{tabular}{|c|c|c|c|c|c|c|c|c|c|}
\hline Variable & \multicolumn{2}{|c|}{ Medias y d.t. } & \multirow{2}{*}{$\frac{\text { FACTOR }}{\text { Aplic. Pre/Post }}$} & \multirow{2}{*}{$\frac{\mathbf{g l}}{1 \mathrm{y} 82}$} & \multirow{2}{*}{$\frac{\text { MC }}{3569.745}$} & \multirow{2}{*}{$\frac{\mathbf{F}}{78.278}$} & \multirow{2}{*}{$\frac{\mathbf{p}}{.000^{* *}}$} & \multirow{2}{*}{$\frac{\text { Potencia }}{-}$} & \multirow{2}{*}{$\begin{array}{c}\begin{array}{c}\text { Eta2 } \\
\text { parcial }\end{array} \\
.474\end{array}$} \\
\hline & $\begin{array}{l}\text { Aplic. pre. } 24.77 \\
\text { (d.t. 6.66) }\end{array}$ & $\begin{array}{c}\text { Aplic. post. } 33.72 \\
\text { (d.t. 7.58) }\end{array}$ & & & & & & & \\
\hline Pensamiento & $\begin{array}{l}\text { G.E. } 30.38 \\
\text { (d.t. 5.26) }\end{array}$ & $\begin{array}{l}\text { G.C. } 28.11 \\
\text { (d.t. } 5.14 \text { ) }\end{array}$ & Grupo Exp/Cont & 1 y 82 & 230.337 & 4.254 & $.042 *$ & - & .047 \\
\hline Crítico Total & $\begin{array}{l}\text { G.E. pre. } 25.31 \\
\text { (d.t. 6.15) } \\
\text { G.C. pre. 24.22 } \\
\text { (d.t. 7.15) }\end{array}$ & $\begin{array}{l}\text { G.E. post. } 35.45 \\
\text { (d.t. } 8.00 \text { ) } \\
\text { G.C. post. } 32.00 \\
\text { (d.t. } 6.71 \text { ) }\end{array}$ & Grupo/Aplicac. & 1 y 82 & 61.88 & 1.357 & $.247^{\mathrm{NS}}$ & .210 & .015 \\
\hline
\end{tabular}

$\mathrm{NS}=$ correlacción no significativa $(\mathrm{p}>.50) \quad *$ Significativo al .05 $\quad * *$ Altamente significativo .01 
Con respecto a los resultados obtenidos en los cinco factores de Pensamiento Crítico según el momento de medida pre-post, se desprende que existen diferencias significativas en todos los factores $\left(F_{R P}(1,82)=54.69, p=.00 ; F_{R D}(1,82)=53.37, p=.00 ; F_{R I}(1,82)=60.78\right.$, $\left.p=.00 ; F_{T D}(1,82)=37.75, p=.00\right)$ excepto en el de solución de problemas $\left(\mathrm{F}_{\mathrm{SP}}(1,82)=.20\right.$, $p=.64)$. Como se preveía, las puntuaciones obtenidas en el pre son inferiores a las conseguidas después de la intervención. En la comparación entre los grupos, encontramos diferencias significativas en consonancia con la intervención únicamente en el factor de razonamiento práctico $\left(F_{R P}(1,82)=6.43, p=.01\right)$ con respecto al resto de factores $\left(F_{R D}(1,82)=1.19, p=\right.$ $\left..27 ; F_{R I}(1,82)=2.35, p=.12 ; F_{T D}(1,82)=.11, p=.73 ; F_{S P}(1,82)=.86, p=.35\right)$. En cuanto a la interacción de los dos niveles, tampoco se dan diferencias significativas en ninguno de los cinco factores $\left(F_{R P}(1,82)=1.82, p=.18 ; F_{R D}(1,82)=1.45, p=.23 ; F_{R I}(1,82)=.21, p=.64\right.$; $\left.F_{T D}(1,82)=.87, p=.35 ; F_{S P}(1,82)=.00, p=.95\right)$. Vemos que, aunque las medias aumentan como resultado de la intervención, la diferencia entre los grupos no es significativa, ambos mejoran su rendimiento por igual (Véase tabla 3).

Tabla 3: Resumen de la significación de los efectos de ambos factores sobre la variable razonamiento práctico, deductivo e inductivo, toma de decisiones y solución de problemas.

\begin{tabular}{|c|c|c|c|c|c|c|c|c|c|}
\hline Variables & \multicolumn{2}{|c|}{ Medias y d.t. } & FACTOR & gl & MC & $\mathbf{F}$ & $\mathbf{p}$ & Potencia & $\begin{array}{c}\text { Eta2 } \\
\text { parcial }\end{array}$ \\
\hline \multirow{3}{*}{$\begin{array}{c}\text { Razonamiento } \\
\text { Práctico }\end{array}$} & $\begin{array}{l}\text { Aplic. pre. } 5.19 \\
\text { (d.t. 2.34) }\end{array}$ & $\begin{array}{l}\text { Aplic. post.7.74 } \\
\text { (d.t. 2.53) }\end{array}$ & Aplic. Pre/Post & 1 y 82 & 290.644 & 54.695 & $.000 * *$ & - & .386 \\
\hline & $\begin{array}{l}\text { G.E. } 6.94 \\
\text { (d.t. 1.66) }\end{array}$ & $\begin{array}{l}\text { G.C. } 6.00 \\
\text { (d.t. 1.83) }\end{array}$ & Grupo Exp/Cont & 1 y 82 & 39,582 & 6,434 & $.013 *$ & - & .069 \\
\hline & $\begin{array}{l}\text { G.E. pre. } 5.43 \\
\text { (d.t. } 2.13) \\
\text { G.C. pre. } 4.96 \\
\text { (d.t. 2.52) }\end{array}$ & $\begin{array}{l}\text { G.E. post. } 8.45 \\
\quad \text { (d.t. 2.20) } \\
\text { G.C. post. } 7.04 \\
\quad \text { (d.t. 2.66) }\end{array}$ & Grupo/Aplicac. & 1 y 82 & 9,700 & 1,825 & $.180^{\mathrm{NS}}$ & .267 & .021 \\
\hline \multirow{3}{*}{$\begin{array}{c}\text { Razonamiento } \\
\text { Deductivo }\end{array}$} & $\begin{array}{l}\text { Aplic. pre. } 3.56 \\
\text { (d.t. 2.08) }\end{array}$ & $\begin{array}{l}\text { Aplic. post. } 5.82 \\
\text { (d.t. 2.34) }\end{array}$ & Aplic. Pre/Post & 1 y 82 & 227.788 & 53.372 & $.000 * *$ & - & .380 \\
\hline & $\begin{array}{l}\text { G.E. } 4.88 \\
\text { (d.t. 1.75) }\end{array}$ & $\begin{array}{l}\text { G.C. } 4.50 \\
\text { (d.t. } 1.57 \text { ) }\end{array}$ & Grupo Exp/Cont & 1 y 82 & 6.642 & 1.192 & $.278^{\mathrm{NS}}$ & .191 & .014 \\
\hline & $\begin{array}{l}\text { G.E. pre. } 3.57 \\
\text { (d.t. } 2.23 \text { ) } \\
\text { G.C. pre. } 3.56 \\
\quad \text { (d.t. } 1.96)\end{array}$ & $\begin{array}{l}\text { G.E. post. } 6.20 \\
\quad \text { (d.t. } 2.31 \text { ) } \\
\text { G.C. post. } 5.44 \\
\quad \text { (d.t. } 2.34 \text { ) }\end{array}$ & Grupo/Aplicac. & 1 y 82 & 6.215 & 1.456 & $.231^{\mathrm{NS}}$ & .222 & .016 \\
\hline \multirow{3}{*}{$\begin{array}{l}\text { Razonamiento } \\
\text { Inductivo }\end{array}$} & $\begin{array}{l}\text { Aplic. pre. } 4.01 \\
\text { (d.t. 1.75) }\end{array}$ & $\begin{array}{l}\text { Aplic. post. } 6.09 \\
\text { (d.t.1.88) }\end{array}$ & Aplic. Pre/Post & 1 y 82 & 192.510 & 60.785 & $.000 * *$ & - & .411 \\
\hline & $\begin{array}{l}\text { G.E.5.26 } \\
\text { (d.t. } 1.31 \text { ) }\end{array}$ & $\begin{array}{l}\text { G.C. } 4.84 \\
\text { (d.t. } 1.25)\end{array}$ & Grupo Exp/Cont & 1 y 82 & 7.734 & 2.350 & $.129^{\mathrm{NS}}$ & .329 & .026 \\
\hline & $\begin{array}{l}\text { G.E. pre. } 4.16 \\
\text { (d.t. } 1.75) \\
\text { G.C. pre. } 3.87 \\
\text { (d.t. } 1.68 \text { ) }\end{array}$ & $\begin{array}{c}\text { G.E. post. } 6.36 \\
\text { (d.t. } 1.90) \\
\text { G.C. post. 5.82 } \\
\text { (d.t. 1.83) }\end{array}$ & Grupo/Aplicac. & 1 y 82 & .690 & .218 & $.642^{\mathrm{NS}}$ & .075 & .002 \\
\hline
\end{tabular}




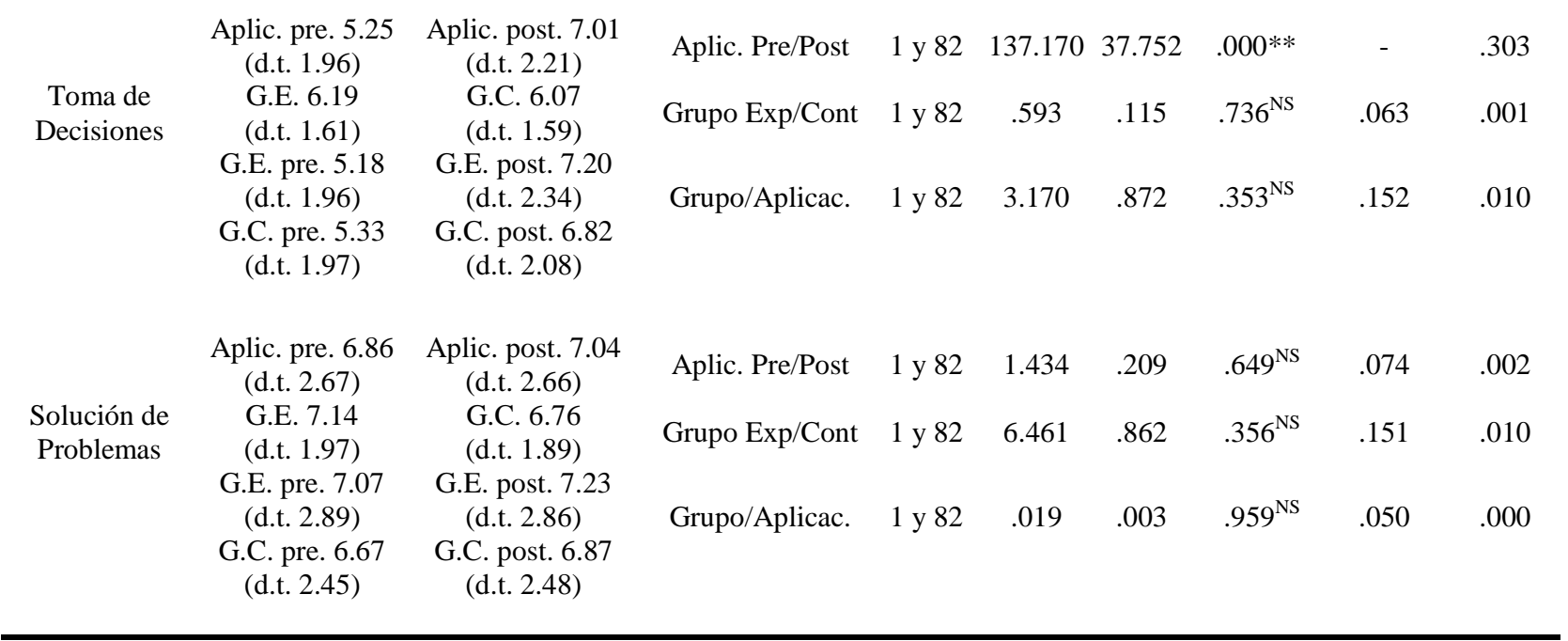

\footnotetext{
$\mathrm{NS}=$ correlacción no significativa $(\mathrm{p}>.50) \quad *$ Significativo al .05 $\quad * *$ Altamente significativo .01
}

Con respecto a la variable motivación total los datos nos muestran que en el factor intragrupal, existen diferencias significativas $p<.05\left(F_{M O}(1,82)=5,99, p=.01\right)$. Esta diferencia se refleja en las medias pero, en este caso, en sentido contrario a lo esperado, ya que las puntuaciones disminuyen en la medida post $\left(M_{\text {Opre }}=66.16 ; M_{\text {Opost }}=64.09\right)$. También se dan diferencias significativas respecto a la relación entre los grupos de intervención y el momento de aplicación pre-post $\left(F_{M O}(1,82)=6.95, p=.01\right)$, donde encontramos que el grupo experimental mantiene sus puntuaciones en ambas medidas $\left(M_{\text {GEpre }}=65.66 ; M_{\text {GEpost }}=65.82\right)$, mientras que el grupo control observa una disminución significativa de las puntuaciones en discordancia con la intervención $\left(M_{G C p r e}=66.67 ; M_{G C p o s t}=62.38\right)$. Sin embargo, a nivel intergrupal no se dan diferencias significativas $\left(F_{M O}(1,82)=.940, p=.33\right)$ (Véase tabla 4$)$.

Tabla 4: Resumen de la significación de los efectos de ambos factores sobre la variable motivación total.

\begin{tabular}{|c|c|c|c|c|c|c|c|c|c|}
\hline Variable & Medi & y d.t. & FACTOR & gl & MC & $\mathbf{F}$ & $\mathbf{p}$ & Potencia & $\begin{array}{c}\text { Eta2 } \\
\text { parcial }\end{array}$ \\
\hline \multirow{3}{*}{$\begin{array}{c}\text { Motivación } \\
\text { Total }\end{array}$} & $\begin{array}{l}\text { Aplic. pre. } 66.16 \\
\text { (d.t. 5.72) }\end{array}$ & $\begin{array}{c}\text { Aplic. post. } 64.09 \\
\text { (d.t. 8.42) }\end{array}$ & Aplic. Pre/Post & 1 y 82 & 189.715 & 5.998 & $.016^{*}$ & - & .065 \\
\hline & $\begin{array}{l}\text { G.E. } 65.73 \\
\text { (d.t. } 6.28)\end{array}$ & $\begin{array}{l}\text { G.C. } 64.52 \\
\text { (d.t. } 5.54)\end{array}$ & Grupo Exp/Cont & 1 y 82 & 65.837 & .940 & $.335^{\mathrm{NS}}$ & .160 & .011 \\
\hline & $\begin{array}{l}\text { G.E. pre. } 65.66 \\
\text { (d.t. } 6.00 \text { ) } \\
\text { G.C. pre. } 66.67 \\
\text { (d.t. } 5.45 \text { ) }\end{array}$ & $\begin{array}{c}\text { G.E. post. 65.82 } \\
\text { (d.t. 8.49) } \\
\text { G.C. post. 62.38 } \\
\text { (d.t. 8.09) }\end{array}$ & Grupo/Aplicac. & 1 y 82 & 220.075 & 6.958 & $.010^{* *}$ & - & .074 \\
\hline
\end{tabular}


Con respecto a los resultados obtenidos en los tres factores de motivación según el momento de medida pre-post, se desprende que no existen diferencias significativas en ninguno de los tres factores $\left(F_{M A}(1,82)=1.46, p=.22 ; F_{M L}(1,82)=3.86, p=.05 ; F_{M R S}(1,82)=\right.$ $1.95, p=.16)$. Las medias de las puntuaciones muestran que se produce una disminución en los factores metas de aprendizaje y metas de logro en discordancia con la intervención y, sin embargo, en el factor metas de refuerzo social se produce un ligero aumento de las puntuaciones, pero no significativo, en consonancia con la intervención. De la misma forma que tampoco se dan diferencias significativas entre los grupos experimental y control $\left(F_{M A}(1,82)=.41\right.$, $\left.p=.52 ; F_{M L}(1,82)=.42, p=.51 ; F_{M R S}(1,82)=1.04, p=.31\right)$, donde las medias de rendimiento son prácticamente similares entre los grupos. Tampoco se dan diferencias significativas en la relación entre la medida pre-post y los grupos de intervención $\left(F_{M A}(1,82)=2.21, p\right.$ $\left.=.14 ; F_{M L}(1,82)=.07, p=.78 ; F_{M R S}(1,82)=1.83, p=.17\right)$ (Véase tabla 5).

\section{Tabla 5: Resumen de la significación de los efectos de ambos factores sobre la variable metas de aprendizaje, de logro y de refuerzo social.}

\begin{tabular}{|c|c|c|c|c|c|c|c|c|c|}
\hline Variables & \multicolumn{2}{|c|}{ Medias y d.t. } & FACTOR & gl & MC & $\mathbf{F}$ & $\mathbf{p}$ & Potencia & $\begin{array}{c}\text { Eta2 } \\
\text { parcial }\end{array}$ \\
\hline \multirow{3}{*}{$\begin{array}{c}\text { Metas de } \\
\text { Aprendizaje }\end{array}$} & $\begin{array}{c}\text { Aplic. pre. 30.65 } \\
\text { (d.t. 3.77) }\end{array}$ & $\begin{array}{c}\text { Aplic. post. 29.95 } \\
\text { (d.t. } 6.12 \text { ) }\end{array}$ & Aplic. Pre/Post & 1 y 82 & 21.692 & 1.469 & $.229^{\mathrm{NS}}$ & .224 & .017 \\
\hline & $\begin{array}{c}\text { G.E. } 30.011 \\
\text { (d.t. 4.77) }\end{array}$ & $\begin{array}{c}\text { G.C. } 30.60 \\
\text { (d.t. } 3.79)\end{array}$ & Grupo Exp/Cont & 1 y 82 & 15.417 & .416 & $.521^{\mathrm{NS}}$ & .098 & .005 \\
\hline & $\begin{array}{l}\text { G.E. pre. } 29.93 \\
\quad \text { (d.t. } 4.08 \text { ) } \\
\text { G.C. pre. } 31.38 \\
\quad \text { (d.t. } 3.32 \text { ) }\end{array}$ & $\begin{array}{l}\text { G.E. post. } 30.09 \\
\text { (d.t. } 6.60) \\
\text { G.C. post. 29.82 } \\
\quad \text { (d.t. 5.69) }\end{array}$ & Grupo/Aplicac. & 1 y 82 & 32.704 & 2.215 & $.140^{\mathrm{NS}}$ & .313 & .025 \\
\hline \multirow{3}{*}{$\begin{array}{c}\text { Metas de } \\
\text { Rendimiento }\end{array}$} & $\begin{array}{l}\text { Aplic. pre. } 25.81 \\
\text { (d.t. 3.42) }\end{array}$ & $\begin{array}{c}\text { Aplic. post. } 24.56 \\
\text { (d.t. 6.16) }\end{array}$ & Aplic. Pre/Post & 1 y 82 & 68.990 & 3.868 & $.052^{\mathrm{NS}}$ & .494 & .043 \\
\hline & $\begin{array}{l}\text { G.E. } 25.46 \\
\text { (d.t. } 4.54)\end{array}$ & $\begin{array}{c}\text { G.C. } 24.91 \\
\text { (d.t. } 3.43)\end{array}$ & Grupo Exp/Cont & 1 y 82 & 13.695 & .423 & $.517^{\mathrm{NS}}$ & .099 & .005 \\
\hline & $\begin{array}{l}\text { G.E. pre. } 26.00 \\
\text { (d.t. } 3.58 \text { ) } \\
\text { G.C. pre. } 25.62 \\
\text { (d.t. 3.29) }\end{array}$ & $\begin{array}{l}\text { G.E. post. 24.93 } \\
\text { (d.t. 6.75) } \\
\text { G.C. post. 24.20 } \\
\text { (d.t. 5.58) }\end{array}$ & Grupo/Aplicac. & 1 y 82 & 1.394 & .078 & $.780^{\mathrm{NS}}$ & .059 & .001 \\
\hline \multirow{3}{*}{$\begin{array}{l}\text { Metas de } \\
\text { Refuerzo } \\
\text { Social }\end{array}$} & $\begin{array}{c}\text { Applic. pre. } 9.69 \\
\text { (d.t. 3.09) }\end{array}$ & $\begin{array}{l}\text { Applic. post. } 10.44 \\
\text { (d.t. 5.47) }\end{array}$ & Applic. Pre/Post & 1 y 82 & 25.011 & 1.950 & $.166^{\mathrm{NS}}$ & .282 & .022 \\
\hline & $\begin{array}{c}\text { G.E. } 10.46 \\
\text { (d.t. 4.05) }\end{array}$ & $\begin{array}{l}\text { G.C. } 9.67 \\
\text { (d.t. 3.19) }\end{array}$ & Group Exp/Cont & 1 y 82 & 27.638 & 1.040 & $.311^{\mathrm{NS}}$ & .172 & .012 \\
\hline & $\begin{array}{l}\text { G.E. pre. } 9.73 \\
\text { (d.t. } 3.22 \text { ) } \\
\text { G.C. pre. } 9.67 \\
\text { (d.t. } 3.00 \text { ) }\end{array}$ & $\begin{array}{l}\text { G.E. post. } 11.20 \\
\text { (d.t. } 6.10) \\
\text { G.C. post. } 9.69 \\
\text { (d.t. } 4.71 \text { ) }\end{array}$ & Group/Applicac. & 1 y 82 & 23.551 & 1.836 & $.179^{\mathrm{NS}}$ & .268 & .021 \\
\hline
\end{tabular}




\section{Discusión}

Los resultados que aporta este estudio ponen de manifiesto que la iniciativa empleada ha sido eficaz, pero con reservas. Respecto a la instrucción en las habilidades de Pensamiento Crítico, hemos obtenido unos resultados de eficacia satisfactorios. Las dimensiones de razonamiento práctico, deducción, inducción y toma de decisiones mejoraron después de la intervención, produciéndose un cambio sustancial en ambos grupos, si bien dándose un rendimiento superior en el grupo experimental debido a la intervención realizada en motivación. Como hemos visto, la intervención en motivación canalizada a través de la utilidad, ayuda a los estudiantes en la promoción hacia una mayor transferencia, lo cual reporta en una mayor integración de los contenidos y, por lo tanto, un mejor rendimiento.

No obstante, creemos que una interpretación de que no se hayan producido mejoras significativas en el factor de solución de problemas descansa en el hecho de que se trató de un bloque temporalmente corto y no permitió una interiorización significativa de los procedimientos. A la par, pensamos que tanto toma de decisiones, como solución de problemas, se trabajaron de un modo semejante en lo referente a estrategias generales. De hecho, es muy complicado separar estas dos estrategias, porque una buena parte de los casos en los que se resuelve un problema se hace a través de una elección. En el futuro estamos trabajando en la dirección de considerar estas dos dimensiones como un caso especial una de otra. En todo caso, estos resultados nos permiten seguir trabajando en la dirección planteada, con el fin de mejorar nuestro trabajo docente y de evaluación.

De esta forma, nuestras hipótesis quedarían comprobadas parcialmente ya que, por una parte, se produce un aumento del rendimiento en las puntuaciones de Pensamiento Crítico gracias a la intervención ARDESOS y, por otra, el grupo experimental obtiene un rendimiento superior al grupo control en algunas de las variables objeto de estudio. Asimismo, concluiríamos que la intervención en motivación es eficaz ya que contribuye a la mejora del rendimiento, pero no lo suficiente como para producir diferencias significativas en todas y cada una de las variables del estudio, especialmente cuando se trata de los resultados obtenidos de la intersección de los niveles intra e intergrupal.

Sin embargo, con respecto a las puntuaciones obtenidas en motivación, observamos en los resultados que, a nivel intragrupal, se dieron diferencias significativas pero en sentido con- 
trario a la hipótesis planteada, es decir, transcurrida la intervención, la media de las puntuaciones de motivación en vez de experimentar un aumento, obtuvo un decremento de dos puntos. A nivel intergrupal, no se consiguieron diferencias significativas, ya que ambos grupos alcanzaron medias análogas. Sin embargo, en la intersección de los dos niveles, sí se lograron diferencias significativas, donde se advirtió que el grupo experimental mantuvo su media en motivación a lo largo de la intervención, mientras que el grupo control la disminuyó significativamente, aún habiendo registrado una puntuación superior (1 punto) respecto al grupo experimental en la medida pre de la intervención; no obstante, este dato no era significativo.

Siendo así, estos datos caminan a favor de la hipótesis planteada. Aunque se esperaba un aumento significativo de las puntuaciones en motivación en el grupo experimental con respecto al grupo control, los resultados nos confirman que, si bien no se logró ese incremento, al menos sí se produjo un mantenimiento de dichas puntuaciones en el grupo experimental. En cambio, en el grupo control que no recibió dicho programa de ayudas motivacionales, se advirtió un descenso en la media de las puntuaciones al término de la intervención. La explicación es clara, el aprendizaje de las habilidades de Pensamiento Crítico es muy costoso y requiere de mucho esfuerzo continuado. Que este aprendizaje demande mucha voluntad hace que si a lo largo de la instrucción, no se retroalimenta la disposición de los alumnos a aprender dichas habilidades, cabe la posibilidad de que las puntuaciones en motivación desciendan, como acabamos de comprobar en estos resultados y, con ello, el rendimiento en Pensamiento Crítico. Por tanto, consideramos que, gracias a la intervención que se lleva a cabo con el grupo experimental, se consigue que la motivación, de no aumentar, se mantenga a lo largo de toda la intervención. Como fácilmente ha podido desprenderse a lo largo de todo este trabajo, cuestión difícil de lograr. Consiguientemente, gracias a dicha intervención, logramos que el rendimiento en Pensamiento Crítico de este grupo, fuera significativamente mayor respecto al grupo control. No obstante, aunque el rendimiento de este grupo es inferior al del grupo experimental, mejora su rendimiento a lo largo de la intervención, cuestión que atribuimos principalmente al contexto curricular.

Por ende, se deduce que la motivación es un constructo que modula el Pensamiento Crítico, pero, por sí misma, no consigue aumentar el rendimiento en las habilidades de Pensamiento Crítico. Como exponíamos al comienzo de este estudio, solamente con las disposiciones obtendríamos un aumento del rendimiento en estas habilidades menor. Y viceversa, sólo interviniendo en Pensamiento Crítico, no lograríamos llegar a tales puntuaciones de eje- 
cución. Efectivamente, ambos factores, disposiciones y habilidades, son necesarios para el buen uso y práctica de dichas habilidades y su transferencia a la vida cotidiana.

Hasta aquí, hemos expuesto lo que concuerda con nuestros pronósticos. Ahora es necesario tratar lo que no se adecúa a nuestros planteamientos, y lo que parece ser fruto de las limitaciones de nuestro procedimiento. Primeramente, la elección de la prueba para medir el constructo de motivación no ha sido la mejor opción que pudiera recoger la esencia que buscábamos en este tipo de intervención. Segundo, nuestro método de instrucción de motivación ha funcionado en buena medida, tal como se aprecia en los resultados ya discutidos. Sin embargo, como suele suceder con los procedimientos de intervención, siempre hay factores que no se controlan de manera conveniente, dada la complejidad de toda situación de aprendizaje, que, además, está en parte condicionada institucionalmente. Es posible que, una vez subsanadas las limitaciones señaladas, como las mencionadas en la dimensión de solución de problemas, algunos resultados no obtenidos, se hubieran podido conseguir. Téngase en cuenta, que el programa de tutorías depende del buen funcionamiento de la instrucción en las habilidades de pensamiento. En estos momentos, estamos considerando estas limitaciones y modificando la instrucción. Al mismo tiempo, estamos revisando todas las pautas seguidas en el procedimiento con el fin de mejorar toda la intervención motivacional. En tercer lugar, pueden haberse producido ciertos efectos negativos en el trabajo en equipo, que hayan tenido como efecto la disminución del rendimiento o del esfuerzo, y que en las tutorías no se hayan solucionado (Salomon y Globerson, 1989). Nuestro método no contempla controlar estas limitaciones del trabajo en equipo. Por ello, es posible que parte de los resultados que no se han obtenido se deban a esta falta de control del trabajo en grupo. En la actualidad, como decíamos, estamos estudiando cómo incorporar controles que eviten estos sesgos. Uno que se ha probado en un estudio ya realizado, es emplear un sistema valoración anónimo del esfuerzo de los miembros del grupo, por cada uno de sus componentes. Esta valoración pondera la calificación de cada miembro del gurpo en cada tarea, de modo que puede mjoerar o emporar su rendimiento, en función de su esfuerzo en el trabajo de grupo. Hay otros aspectos que estamos considerando para futuras investigaciones. 


\section{Referencias}

APA (1990). Critical thinking: A statement of expert consensus for purposes of educa- tional assessment and instruction. Executive Summary "The Delphi Report". http://www.insightassessment.com/dex.html.

Eccles, J., Adler, T. F., Futterman, R., Goff, S. B., Kaczala, C. M., Meece, J. L. \& Midgley, C. (1983). Expectancies, values, and academic behaviors. In J. T. Spence (Ed.), Achievement and achievement motivation (pp. 75-146). San Francisco, CA: W. H. Freeman.

Eccles, J. (1987). Gender roles and women's achievement-related decisions.Psychology of Women, 11, 135-172. DOI: 10.1111/j.1471-6402.1987.tb00781.x.

Eccles, J. (1993). School and family effects on the ontogeny of children's interests, self-perceptions and activity choice. In J. Jacobs (Ed.), Nebraska Symposium on Motivation, 1992: Developmental perspective on motivation (pp.145-208). Lincoln, NE: University of Nebraska Press.

Eccles, J., Adler, T. F., Futterman, R., Goff, S. B., Kaczala, C. M. \& Meece, J. L. (1983). Expectancies, values and academic behaviors. Achievement and achievement motivation (pp. 75-146). San Francisco, CA: W.H. Freeman.

Eccles, J. \& Wigfield, A. (2002). Motivational beliefs, Values and Goals. In S.T. Fiske, D.L. Schacter y C. Sahn-Waxler (eds.), Annual Review of Psychology (pp. 109-132). Palo Alto, CA: Annual Reviews.

Ennis, R. H. (1996). Critical Thinking. Upper Saddle River, Nueva Jersey: Prentice-Hall.

Facione, P. A. (2011). Think critically. New York: Prentice Hall.

Halpern, D. F. (1998). Teaching critical thinking for transfer across domains - dispositions, skills, structure training, and metacognitive monitoring. American Psychologist, 53 (4), 449-455. DOI: 10.1037/0003-066x.53.4.449.

Halpern, D. F. (2003). Thought and knowledge: An introduction to critical thinking (Fourth edition). Hillsdale, NJ: Erlbaum. 
Paul, R. W. \& Elder, L. (2001). Critical thinking: Tools for taking charge of your learning and your life. Upper Saddle River, NJ: Prentice Hall.

Rinaudo, M. C., Barrera, M. L. y Donolo, D. S. (2006). Motivación para el aprendizaje en alumnos universitarios. Revista Electrónica de Motivación y Emoción, 22 (9), 84-93. http://reme.uji.es/reme/numero22/indexsp.html.

Rivas, S. F. y Saiz, C. (2012). Validación y propiedades psicométricas de la prueba de pensamiento crítico PENCRISAL. Revista Electrónica de Metodología Aplicada. Vol. $17(1), 18-34$.

Saiz, C. (2002). Solución de problemas. En C. Saiz (Ed.), Pensamiento crítico: conceptos básicos y actividades prácticas (p. 183-211). Madrid: Pirámide.

Saiz, C. y Nieto, A. M. (2002). Pensamiento crítico: capacidades y desarrollo. En C. Saiz (Ed.), Pensamiento crítico: conceptos básicos y actividades prácticas (p.15-19). Madrid: Pirámide.

Saiz, C. y Rivas, S. F. (2008). Intervenir para transferir en pensamiento crítico. Praxis, 10 (13), 129-149.

Saiz, C. y Rivas, S. F. (2008). Evaluación en pensamiento crítico: una propuesta para diferenciar formas de pensar. Ergo, Nueva Época, 22-23, 25-26.

Saiz, C. \& Rivas, S. F. (2011). Evaluation of the ARDESOS program: an initiative to improve critical thinking skills. Journal of the Scholarship of Teaching and Learning, 11 (2), 34-51.

Salomon, G. \& Globerson, T. (1989). When teams do not function the way ought to. International Journal of Educational Reseach, 13, 89-99. DOI: 10.1016/08830355(89)90018-9.

Wigfield, A. (1994). Expectancy-value theory of achievement motivation: A developmental perspective. Educational Psychology Review, 6, 49-78. DOI:10.1007/BF02209024.

Wigfield, A. \& Eccles, J. (1992). The development of achievement task values: A theoretical analysis. Developmental Review, 12, 265-310. http://dx.doi.org/10.1016/02732297(92)90011-P. 
Wigfield, A. \& Eccles, J. (2000). Expectancy-Value Theory of Achievement Motivation. $\begin{array}{llll}\text { Contemporary educational } & \text { 6sychology, }\end{array}$ http://dx.doi.org/10.1006/ceps.1999.1015. 